JOURNAL OF SYNCHROTRON RADIATION

ISSN 1600-5775

Received 30 April 2021

Accepted 24 July 2021

Edited by S. Svensson, Uppsala University, Sweden

Keywords: X-ray optics; high-heat-load optics; multilayer monochromator; finite-element analysis.

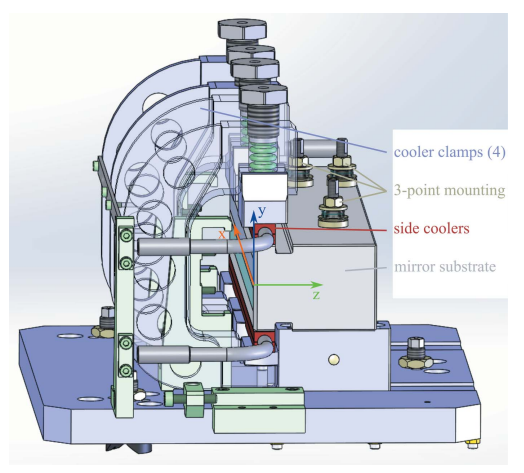

\section{Thermal optimization of a high-heat-load double-multilayer monochromator}

\author{
Philipp Brumund,* Juan Reyes-Herrera, Christian Morawe, Thomas Dufrane, \\ Helena Isern, Thierry Brochard, Manuel Sánchez del Río and Carsten Detlefs
}

ESRF - The European Synchrotron, 38043 Grenoble Cedex 9, France. *Correspondence e-mail: brumund@esrf.fr

Finite-element analysis is used to study the thermal deformation of a multilayer mirror due to the heat load from the undulator beam at a low-emittance synchrotron source, specifically the ESRF-EBS upgrade beamline EBSL-2. The energy bandwidth of the double-multilayer monochromator is larger than that of the relevant undulator harmonic, such that a considerable portion of the heat load is reflected. Consequently, the absorbed power is non-uniformly distributed on the surface. The geometry of the multilayer substrate is optimized to minimize thermally induced slope errors. We distinguish between thermal bending with constant curvature that leads to astigmatic focusing or defocusing and residual slope errors. For the EBSL-2 system with grazing angles $\theta$ between 0.2 and $0.4^{\circ}$, meridional and sagittal focal lengths down to $100 \mathrm{~m}$ and $2000 \mathrm{~m}$, respectively, are found. Whereas the thermal bending can be tuned by varying the depth of the 'smart cut', it is found that the geometry has little effect on the residual slope errors. In both planes they are $0.1-0.25 \mu \mathrm{rad}$. In the sagittal direction, however, the effect on the beam is drastically reduced by the 'foregiveness factor', $\sin (\theta)$. Optimization without considering the reflected heat load yields an incorrect depth of the 'smart cut'. The resulting meridional curvature in turn leads to parasitic focal lengths of the order of $100 \mathrm{~m}$.

\section{Introduction}

The emittance of synchrotron radiation sources in fourthgeneration and upgraded storage rings is much lower than at third-generation sources, in particular in the horizontal direction (Tavares et al., 2018; Biasci et al., 2014; Raimondi, 2016). Consequently the requirements for beamline optics are more strict in terms of tolerances, aberrations, surface and heat load errors than in the existing optical designs and have to be re-evaluated and optimized. Here we analyze the thermal deformation of a high-heat-load double-multilayer monochromator (DMM) designed for the ESRF upgrade beamline EBSL2, to be located at ID03.

Multilayer mirrors (MLs) may have different roles at the beamline. They can be used as focusing elements (e.g. Kirkpatrick-Baez mirrors), monochromators (to select a wide or pink energy spectrum out of a white primary beam), highheat-load elements (to reduce the power transferred to downstream optics) and harmonic rejectors (Susini, 1995; Ziegler, 1995; Morawe, 2019). The planned EBSL2 DMM will make use of all of these functions, except focusing, although (as will be shown later) the thermal deformations may induce a parasitic focusing or defocusing that must be considered in the global scheme of the beamline.

The performance of reflecting optics depends critically on the quality of the mirror surface. Nowadays, the limiting factors of most synchrotron beamlines are the surface errors in 
the optics. They have several origins: (i) surface finish introduced during manufacturing (slope errors, height errors), (ii) optical aberrations (shape errors), (iii) shape imperfections induced by strain due to mechanical mounting ('clamping'), and (iv) shape and slope errors due to thermal deformations arising from the high heat absorbed by the optical element. In the grazing-incidence geometry used in most reflecting optics for hard X-rays, the sagittal slope errors have a smaller effect on the beam degradation than meridional slope errors (DiGennaro et al., 1988; de Castro \& Reininger, 1991). The 'foregiveness factor' $1 / \sin (\theta)$ is particularly large in multilayer and total reflectivity mirrors, as $\theta$ (the grazing angle of incidence) is small [typically $<2^{\circ}$ for hard $\mathrm{X}$-rays (Morawe et al., 2001)].

Here, we analyze the thermal deformations and discuss some solutions to minimize their effect. Compared with previous studies (Khounsary, 1999; Mattenet et al., 2001; Zhang et al., 2013; Knopp et al., 2018), our DMM receives a higher heat load and higher power density. Furthermore, due to the low emittance of the ESRF-EBS storage ring, the spectrum of the undulator source has narrower peaks, which can be fully reflected by the ML. This leads to a non-uniform power density on the multilayer surface which must be taken into account.

The heat load induces two distinct types of deformation: (i) global shape errors that can be approximated by a constant curvature, which have a focusing or defocusing effect that can be corrected with downstream optics (called 'systematic shape errors'), and (ii) residual shape and slope errors superposed on these systematic errors and cannot be easily corrected by usual focusing devices (called 'residual errors').

The paper is organized as follows. Section 2 summarizes the concept and history of the chosen multilayer mirror design, a thick silicon substrate, cooled at the sides, with a channel or 'notch' at each side whose geometry is optimized to minimize the thermal deformation. The mirror will deflect in the horizontal plane. Section 3 describes the main elements of the beamline considered in the simulations: source, slits and attenuators, and multilayer coating. Section 4 describes the details of the finite-element analysis (FEA) procedure and parameters used. The results are shown in Section 5 for both meridional and sagittal directions. They are fully discussed in Section 6. Finally, Section 7 summarizes the work and outlines the main conclusions.

\section{State of the art in thermal design}

In the 1990s, extensive research was carried out to reduce the thermal distortions of X-ray mirrors and multilayer optics, as high-heat-load optics were expected to be the bottleneck to exploit third-generation synchrotron X-ray beams (Menthel et al., 1992; Mattenet \& Marot, 1996; Khounsary \& Yun, 1996). In view of future trends of higher heat loads from new, more powerful undulators with decreasing gaps and increasing storage ring currents (Khounsary, 1999), these concerns remain.
Khounsary \& Yun (1996) systematically differentiate three main contributions to the thermal slope errors of X-ray mirrors:

(i) Thermal bending with a main curvature component resulting from a temperature gradient along the mirror depth.

(ii) Thermal 'mapping' that is linked to local thickening of the ML substrate due to thermal expansion that occurs under varying absorbed heat load over it's surface (related to the spatial variation of the power density).

(iii) Ripple distortions in the case of internal cooling channels. Most modern designs (including the one discussed here) use indirect cooling, and therefore do not exhibit ripple distortions.

The same authors developed the concept of top-side cooling with notches ('smart cuts') to control the effective thermal bending moment (Khounsary, 1999; Li et al., 2004; Zhang et al., 2013). With this method the thermal bending moment caused by the hot beam footprint is practically eliminated, under the condition that the heat load is constant along the length of the mirror. Today, this strategy is followed in most thermal designs for X-ray mirrors. The cross section of a typical X-ray mirror of this type is shown in Fig. 1 with overlaying temperature isolines and geometrical key dimensions.

The main idea of the 'smart cut' is to control and minimize the thermal bending moment $M_{\mathrm{T} y}$ around the $y$-axis (Boley \& Weiner, 1997; Zhang et al., 2013) which is defined as

$$
\begin{aligned}
M_{\mathrm{T} y}(x) & =\int_{A} \alpha E T(x, y, z)\left(z-z_{n}\right) \mathrm{d} A \\
& =\alpha E \int_{0}^{d} \underbrace{\left[\int_{y_{0}}^{y_{1}} T(x, y, z) \mathrm{d} y\right]}_{I T(x, z)}\left(z-z_{\mathrm{n}}\right) \mathrm{d} z,
\end{aligned}
$$

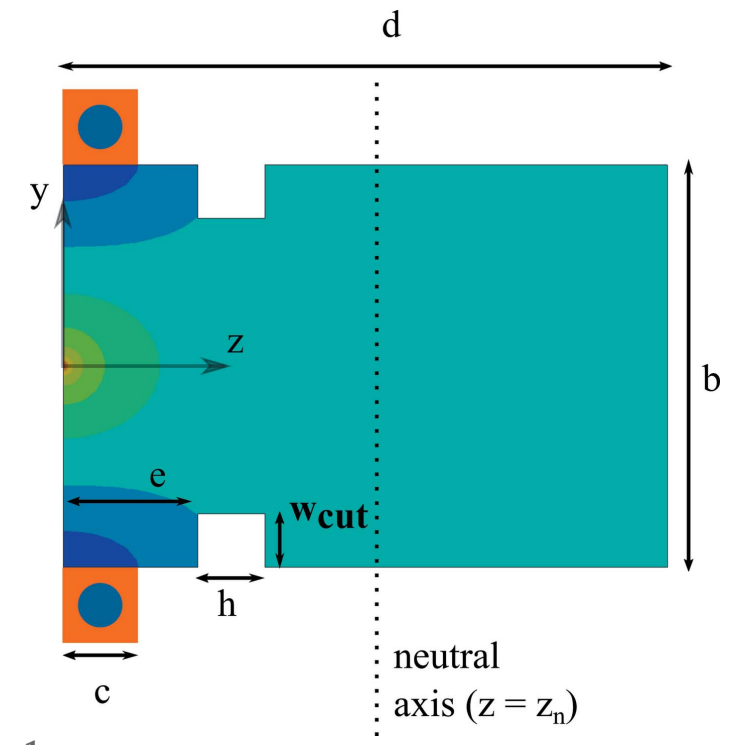

Figure 1

Cross section of a typical mirror substrate showing the stationary temperature distribution resulting from heating the optical surface $(x, y, z=0)$ with the $\mathrm{X}$-ray beam and cooling the upper sides. The indirectly cooling side-coolers are shown in orange. The relevant geometrical design parameters are also shown. Adapted from Khounsary (1999) with permission. 
where $\alpha$ is the linear coefficient of thermal expansion, $E$ the Young's modulus, $T(x, y, z)$ the local temperature at position $(x, y, z)$, and the integral is taken over the cross section $A$ of the mirror. The origin of the coordinate system is in the center of the reflecting surface. $z_{\mathrm{n}}$ identifies the neutral axis, i.e. the centroid of the cross section. In the case of a rectangular cross section, $z_{\mathrm{n}}=d / 2$. In cases with large temperature differences, the temperature dependence of $\alpha$ must be taken into account.

The thermal bending moment is linked to the thermal deformations $w(x)$ in the $z$-direction ${ }^{\mathbf{1}}$ of the optics surface by beam theory and a second-order differential equation,

$$
\frac{\partial^{2} w(x)}{\partial x^{2}}=\kappa(x)=-\frac{M_{\mathrm{T} y}(x)}{E I_{y}},
$$

where $\kappa(x)=1 / R(x)$ denotes the local curvature with radius $R(x)$, and $I_{y}$ the second moment of inertia around the $y$-axis. As noticed by Zhang et al. (2013) for a rectangular crosssection and constant heat profile along the mirror $(x)$, the thermal bending moment [equation (3)] vanishes if the integral $I T(z)=\int T(x, y, z) \mathrm{d} y=$ const, i.e. does not vary over the depth $(z)$ of the mirror. ${ }^{2}$

This is exploited by side-cooling near the top of the mirror in combination with the 'smart cuts' (of depth $w_{\text {cut }}$ in Fig. 1) that isolate the cool regions and prevent over-cooling of the bottom part of the substrate. When furthermore the heat load is constant along the length $(x)$ of the mirror, the geometry can be optimized such that the only remaining slope errors are linked to end effects (Zhang et al., 2013), also visible in Fig. 9. To date, this method has been used to minimize the total r.m.s. slope error, not distinguishing between a mean curvature (due to the bending moment) and residual slope errors (thermal mapping).

As mentioned previously by Khounsary (1999) and Zhang et al. (2013), the bending moment results in a varying curvature $\kappa(x)$ as shown in equation (3), that can be decomposed into an average curvature $\kappa$ superposed with a residual slope error $\Delta \alpha(x)$. The average curvature leads to an effective focusing or defocusing of the reflected beam. ${ }^{3}$ This focusing effect has already been investigated for use in thermal benders, with promising results (Mattenet et al., 2001). Such systematic shape errors (and the resulting slope errors) can be corrected with downstream optics, e.g. a second downstream bendable mirror (Knopp et al., 2018).

Residual slope errors after the subtraction of the main curvature cannot be corrected with simple focusing or defo-

\footnotetext{
${ }^{\mathbf{1}}$ For consistency with the existing literature (Boley \& Weiner, 1997; Zhang et al., 2013), we use $w(x)$ for the height displacement due to bending and thermal deformation of the mirror, and $w_{\text {cut }}$ for the depth of the smart cut.

${ }^{2}$ The condition that $I T(z)=$ const is sufficient, but not necessary. In the case of a cross section that is symmetric with respect to the neutral axis $z=z_{\mathrm{n}}$ of the mirror, any function that is also symmetrical with respect to the neutral axis will have a vanishing thermal bending moment. For the more general case of a non-rectangular cross section (such as in Fig. 1) or non-constant integral $I T(z)=f(z)$, the goal of vanishing thermal bending can be achieved if the sum of local thermal strains above the neutral mirror axis are balanced by thermal strains below the neutral axis.

${ }^{3}$ Curved mirrors are extensively used for focusing synchrotron and laboratory X-ray beams (Susini, 1995; Willmott, 2019).
}

cusing optics. More complex adaptive optics can be envisaged (Cocco et al., 2020; Sanchez del Rio et al., 2020).

One of the main goals of this design study is therefore to quantify the systematic and residual slope errors, and to compare them with typical values of polishing errors [50$160 \mathrm{nrad}$ (Vivo \& Barrett, 2021)] and distortions from clamping [ 50-100 nrad (Chareyre \& Brumund, 2021; Vivo \& Barrett, 2021)].

\section{Design specifications}

The DMM system described here will be used on the ESRF upgrade beamline EBSL-2, an upgrade of the existing hard X-ray microscopy instrument on ID06 (Kutsal et al., 2019). It will operate at photon energies between 12 and $60 \mathrm{keV}$. The main experimental technique employed will be dark-field X-ray microscopy (Simons et al., 2015; Yildirim et al., 2020) for three-dimensional (3D) mapping of lattice strain and orientation in crystalline matter. The instrument will furthermore allow pre-characterization of samples at larger length scales using 3D X-ray diffraction (3DXRD; Poulsen, 2012) or diffraction contrast tomography (DCT; Ludwig et al., 2009), such that a region of interest such as a single grain can be selected for high-resolution studies without the need to dismount the sample.

These techniques require uniform, stable illumination of the sample over several hours with minimal angular drift. The beamline does not require fast energy scanning, with the associated rapid changes of the heat load due to changes of the undulator settings and Bragg angle.

\subsection{Source}

The selected source device is a $2 \mathrm{~m}$-long cryogenically cooled in-vacuum undulator with period $16 \mathrm{~mm}$ (CPMU16) and minimum gap $4 \mathrm{~mm}(K=2.08)$. Heat-load calculations were performed for the new ESRF-EBS storage ring. The electron beam at the center of the straight section (where the undulator is placed) has spatial dimensions $\sigma_{\mathrm{h}}=32.2 \mu \mathrm{m}, \sigma_{\mathrm{v}}=$ $3.6 \mu \mathrm{m}$ and divergences $\sigma_{\mathrm{h}^{\prime}}=4.37 \mu \mathrm{rad}, \sigma_{\mathrm{v}^{\prime}}=1.37 \mu \mathrm{rad}$. At the minimum gap $g=4 \mathrm{~mm}$ the planned CPMU16 emits a total power of $17.6 \mathrm{~kW}$. A large fraction of this power is absorbed by apertures in the front-end and by a $400 \mu \mathrm{m}$-thick diamond window. The maximum beam size incident on the DMM is $2 \mathrm{~mm}(\mathrm{~h}) \times 1 \mathrm{~mm}(\mathrm{v})$, with a maximum power of $978 \mathrm{~W}$ and a peak power density of $540 \mathrm{~W} \mathrm{~mm}^{-2}$.

Thermo-mechanical calculations for the ML are carried out for a variety of parameters. For that, different source configurations (mainly different $K$ values) are chosen. They are listed in Table 1. The first six cases correspond to a selection of a combination of both typical working points and high-heatload cases (smallest undulator gap, highest grazing angle). In particular, we expect that load case 3 (working energy $20.25 \mathrm{keV}$ ) will be used for the majority of DFXM experiments, whereas load cases 5 and 6 will be used for 3DXRD characterization experiments. The last case (7) is the worstcase scenario (largest angle and highest power density) that 
Table 1

Selected source working points as load cases (LC) for further investigation.

These load cases cover a wide spectrum of different energies $E$ and used harmonics $n$ at which the DMM will be used. The corresponding undulator $K$ values for the CPMU16 and the power $P_{\text {in }}$ incident on the first ML mirror are stated.

\begin{tabular}{|c|c|c|c|c|c|}
\hline LC & $E(\mathrm{keV})$ & $K$ & $n$ & $P_{\text {in }}(\mathrm{W})$ & Comment \\
\hline 1 & 12.0 & 1.24 & 1 & 642.9 & Minimum energy \\
\hline 2 & 17.0 & 0.72 & 1 & 255.6 & Current main working energy \\
\hline 3 & 20.3 & 2.08 & 3 & 513.2 & Third harmonic minimum gap \\
\hline 4 & 30.0 & 1.51 & 3 & 491.3 & Alternative main working energy \\
\hline 5 & 34.0 & 2.07 & 5 & 495.3 & Fifth harmonic minimum gap \\
\hline 6 & 60.0 & 1.73 & 7 & 393.6 & Maximum energy \\
\hline 7 & 20.3 & 2.08 & & 977.8 & $\begin{array}{l}\text { Worst case (highest grazing angle, } \\
\text { smallest undulator gap } \\
\text { - highest heat load on mirror) }\end{array}$ \\
\hline
\end{tabular}

would lead to the highest substrate temperature. This case does not occur in normal operation, but is considered to ensure that the multilayer optic cannot be damaged by user errors.

\subsection{Beamline configuration}

The relevant beamline components are shown in Fig. 2. The corresponding distances from the source and relevant dimensions are listed in Table 2.

The source consists of a cryogenic permanent in-vacuum undulator with period length $\lambda=16 \mathrm{~mm}$ as described in Section 3.1.

Two beam-defining apertures (BDAs), at $22.3 \mathrm{~m}$ $(2 \mathrm{~mm} \times 30 \mathrm{~mm})$ and $23 \mathrm{~m}(30 \mathrm{~mm} \times 1 \mathrm{~mm})$ from the source, 'cut' the beam to the maximum size required by the downstream optics. These BDAs remove $14.4 \mathrm{~kW}$ of beam power without loss of the useful central cone insertion device (ID) radiation. This drastically decreases the heat load on downstream optical elements.

A $400 \mu \mathrm{m}$-thick diamond window isolates the beamline vacuum system from the storage ring. Furthermore, it absorbs unwanted low-energy radiation (predominantly from the central cone of the ID's first harmonic, which is not important for the experiments), further reducing the beam power by $300 \mathrm{~W}$.

The beam size incident on the DMM is defined by adjustable primary slits (PS) at $27 \mathrm{~m}$ from the source. The hori-

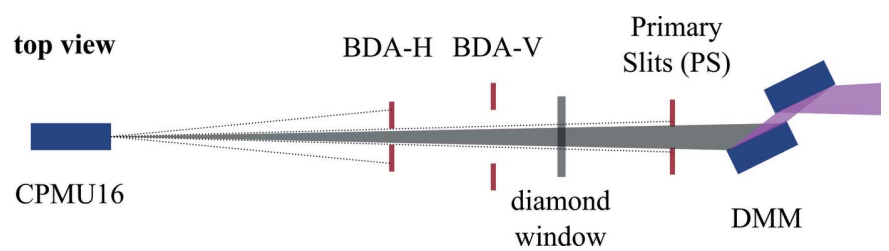

Figure 2

Beamline configuration view showing all relevant components for this calculation: the cryogenic permanent magnet undulator CPMU16, beamdefining apertures (BDA) in the front-end, the front-end diamond window, primary slits and the double multilayer monochromator (DMM). The parameters of the different components are listed in Table 2.
Table 2

Relevant components and corresponding distances on ID03.

\begin{tabular}{|c|c|c|c|}
\hline $\begin{array}{l}\text { Distance } \\
(\mathrm{m})\end{array}$ & Component & $\begin{array}{l}\text { Dimensions } \\
(\mathrm{mm}) \dagger\end{array}$ & Description \\
\hline 0.0 & Undulator & & $\begin{array}{l}\text { Cryogenic permanent magnet } \\
\text { undulator, } L=2 \mathrm{~m}, K_{\max }=2.08 \\
\left(g_{\min }=4 \mathrm{~mm}\right)\end{array}$ \\
\hline 22.3 & BDA-H & $2 \times 30$ & Horizontal beam-defining aperture \\
\hline 23.0 & BDA-V & $30 \times 1$ & Vertical beam-defining aperture \\
\hline 23.5 & Filter & & Diamond filter, $t=0.4 \mathrm{~mm}$ \\
\hline 27.0 & PS & $H \times 1$ & $\begin{array}{l}\text { Primary slits, variable opening in } \\
\text { horizontal direction }\end{array}$ \\
\hline 31.0 & DMM & & $\begin{array}{l}\text { Double multilayer monochromator, } \\
\qquad L=300 \mathrm{~mm}\end{array}$ \\
\hline
\end{tabular}

$\dagger$ Here and below all values are given as (horizontal $\times$ vertical).

zontal opening of the PS is set to the projected size of the DMM's first substrate, $L \sin (\theta)$, in order to provide a homogeneous heat load on the full length of the ML ('overfilling'; see Zhang et al., 2013).

The main subject of this paper, the DMM, is located $31 \mathrm{~m}$ downstream of the source. The beamline components placed downstream of the DMM will be similar to the prototype instruments on ID06 (Kutsal et al., 2019), but are not relevant for this work.

\subsection{DMM geometry and mechanical design}

Based on previous experience at the ESRF, we opted for a DMM with horizontal scattering plane. This offers several advantages:

(i) In the past (including the ESRF-1 storage ring before the EBS upgrade), horizontally scattering mirrors were used to avoid interference 'stripes' that are visible in the vertical direction due to the much longer vertical coherence length. Due to the 'forgiveness factor', meridional slope errors affect the beam much more strongly than sagittal ones. It was therefore advantageous to align the much larger horizontal source size with the meridional direction of the multilayer, i.e. to design the DMM for horizontal scattering.

(ii) Furthermore, horizontal scattering allows for better mechanical stability, with reduced sensitivity to ground vibrations which (at the ESRF) are predominantly vertical. ${ }^{4}$ 'Gravity sag' (Susini, 1995) is also not a problem in horizontal geometry.

At the upgraded ESRF-EBS storage ring, the horizontal source size $\left(\sigma_{\mathrm{h}}=32.2 \mu \mathrm{m}\right)$ is much smaller than at ESRF-1 $\left(\sigma_{\mathrm{h}}=415 \mu \mathrm{m}\right.$ for a high- $\beta$ straight section, as used by ID06), but still larger than the vertical $\left(\sigma_{\mathrm{v}}=3.6 \mu \mathrm{m}\right)$. It is therefore still advisable to align the meridional slope errors horizontally (Brumund et al., 2021).

We therefore chose as a starting point for the design process an existing design [UPBL4 double-mirror with G-bench support, as described by Baker et al. (2013)], which is used on several ESRF beamlines, including ID16, ID15 and ID27. This

\footnotetext{
4 To good approximation only differential movements of the two mirror surfaces matter, as a rigid-body translation of a double mirror does not affect the position or angle of the exit beam.
} 


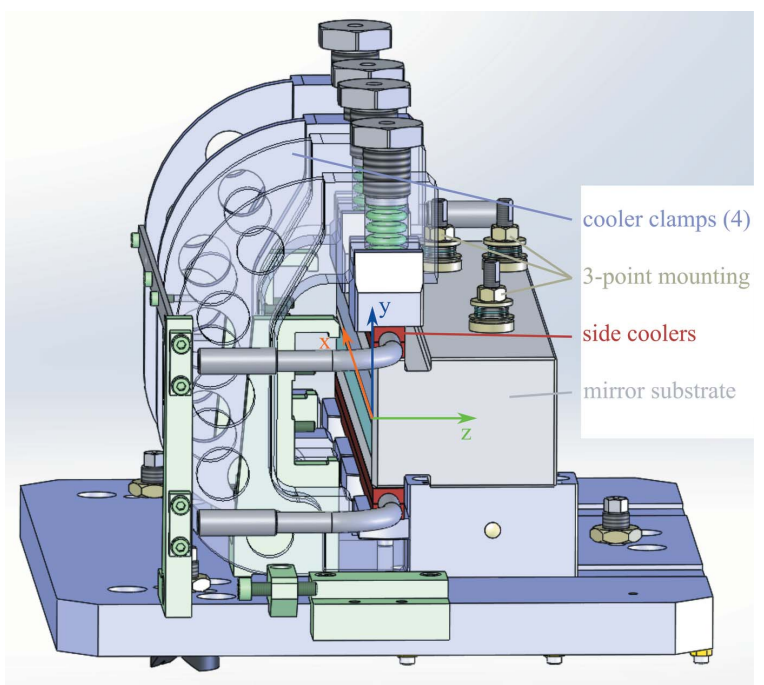

Figure 3

View of a typical ESRF ML system in mechanical assembly. The coolers (red) are clamped to the mirror sides with four cooler clamps. A typical optimized notch is visible just right of the coolers. Mounting to the base is realized with a three-point mount. The presented mirror is the one soon to be installed on the new ESRF EBSL-BL ID27 and has two stripes on its optical face.

design follows the state-of-the-art as discussed above. In particular, the first substrate is top-side cooled, has the 'smart cut' shape shown in Fig. 1, and is 'overfilled' by the incident beam (note that the DMM is reflecting in the horizontal plane, such that the 'top' side in Fig. 1 is pointing to the left in Fig. 3). The system is water-cooled such that the base temperature is approximately $22^{\circ} \mathrm{C}$.

\subsection{Multilayer design}

The parameters of the multilayer coatings and the dimensions of the ML substrates are obtained after a multi-parameter optimization process. The length $L$ of the ML substrate is determined by the useful beam size at the smallest grazing angle $\theta_{\min }\left(L=H / \sin \theta_{\min }\right)$. In our case, $H=1 \mathrm{~mm}$. For a given ML $d$-spacing $\Lambda$, the angle $\theta_{\text {min }}$ is determined by the highest photon energy utilizing this corresponding ML stripe. The choice of ML $d$ spacings(s) thus affects the range of grazing angles and vice versa.

A lower global cost, better optical surface finish and reduced system size favor a short mirror and large $\theta_{\min }$. However, an upper limit of $\theta_{\max }$ is imposed by the power density projected on the substrate, which must be low enough to keep the substrate temperature below $\sim 100^{\circ} \mathrm{C}$ in the worst case (minimum undulator gap, primary slits fully opened and DMM at the largest angle of incidence).

Again, previous experience at ESRF showed that $L=$ $300 \mathrm{~mm}$ with $\theta_{\min }=0.2^{\circ}$ and $\theta_{\max }=0.4^{\circ}$ is a good compromise for the DMM design. In order to cover the entire photon energy range $12-60 \mathrm{keV}$, the substrate will be coated with three parallel ML stripes with parameters listed in Table 3. The coatings are optimized with respect to their peak reflectance, because the ML bandwidth will be larger than the undulator bandwidth. Appropriate material pairs are selected
Table 3

Parameters for the three different multilayer coatings of the DMM.

\begin{tabular}{llll}
\hline Stripe number & 1 & 2 & 3 \\
\hline Period $\Lambda(\AA)$ & 8.50 & 4.80 & 3.05 \\
Absorber, $t_{\text {absorber }}(\AA)$ & $\mathrm{Pd}(3.50)$ & $\mathrm{W}(2.00)$ & $\mathrm{W}(1.45)$ \\
Spacer, $t_{\text {spacer }}(\AA)$ & $\mathrm{C}(5.00)$ & $\mathrm{B}_{4} \mathrm{C}(2.80)$ & $\mathrm{B}_{4} \mathrm{C}(1.60)$ \\
Number of periods & $N=15$ & $N=30$ & $N=70$ \\
Incident angles & & $\theta \simeq 0.40-0.20^{\circ}$ & \\
Energy range $(\mathrm{keV})$ & $12-24$ & $20-40$ & $30-60$ \\
$R^{2}($ peak) & $82-93 \%$ & $66-87 \%$ & $72-88 \%$ \\
$\Delta E / E$ FWHM & $12 \%$ & $6.0-6.9 \%$ & $2.5-2.6 \%$ \\
\hline
\end{tabular}
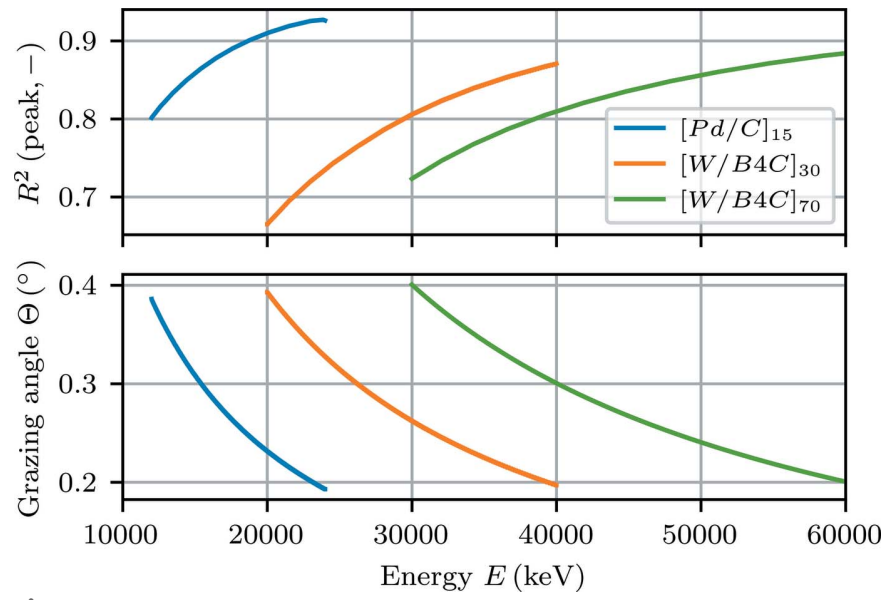

Figure 4

Calculated peak reflectance $R^{2}$ (peak) after two reflections (top) and grazing angle $\theta$ (bottom) of all three ML stripes as indicated by the color code. The calculations assume an r.m.s. surface roughness of $0.3 \mathrm{~nm}$.

to avoid absorption edges in the working energy range. In addition, physical and chemical stability of the layered stacks are required. Each individual stripe allows a different range of photon energies to be covered using grazing angles $\theta$ in the range $0.2-0.4^{\circ}$ (Fig. 4). The multilayers are made by periodic bilayers (of thickness $\Lambda$ ) composed of a layer of a heavy element (absorber) of thickness $t_{\text {absorber }}$ and a layer of a light compound (spacer) of thickness $t_{\text {spacer }}$. The total number of ML periods and the filling factor $\Gamma=t_{\text {absorber }} / \Lambda$ are chosen such that the stress remains below critical limits while maintaining decent peak reflectance levels after two reflections. Moderate annealing at about $150^{\circ} \mathrm{C}$ for a few hours after each coated single stripe will be applied to relax part of the stress. The calculated peak reflectances after two reflections and the corresponding grazing angles are shown in Fig. 4.

\section{Thermo-mechanical simulations}

To accurately estimate the thermal deformation of the mirror substrates we first have to compute the map of power deposited on the substrate's surface (presented in Section 4.1). This distribution is then used as input for thermo-mechanical FEA (Section 4.2).

\subsection{Non-uniform heat load due to reflected power}

ML-based optics can reflect a significant portion of the incident beam power, due to the wide bandwidth $\Delta E / E$. 


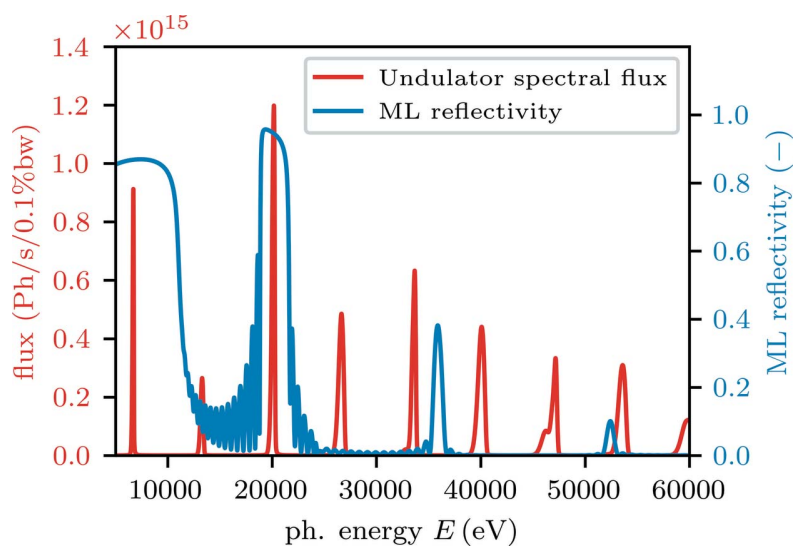

Figure 5

Undulator spectrum (flux on left $y$-axis) and multilayer reflectivity (right $y$-axis) for load case $3(20.25 \mathrm{keV}$, undulator $K=2.08$ at gap $g=4 \mathrm{~mm}$ ), multilayer stripe 1 at $\theta=0.245^{\circ}$, spectrum on aperture $H=1.28 \mathrm{~mm} \times V=$ $1 \mathrm{~mm}$ at $d=31 \mathrm{~m}$ from the source. The multilayer is set to reflect mainly the third undulator harmonic. The diamond window in the frond-end is considered, attenuating the first harmonic. Note that the undulator fundamental falls within the total reflectivity of the multilayer, i.e. low photon energies need to be filtered out.

Figure 5 compares the ML's reflectivity (blue) to the undulator spectrum (red) for load case 3 (see Table 1). Within the central cone, the third undulator harmonic (red) has a much smaller bandwidth than the fundamental ML Bragg peak. Therefore, all radiation from this harmonic is reflected and does not contribute to the thermal load. However, the undulator radiation has a strong dispersion, i.e. the intensity and spectrum vary as a function of the emission angle, such that towards the up- and downstream edge of the ML only a very small portion of the undulator heatload is reflected. Consequently, the local absorbed power density varies over the ML's surface.

The flux shown in Fig. 5 is calculated using an aperture of $1.28 \mathrm{~mm} \times 1 \mathrm{~mm}$ at a distance $d=31 \mathrm{~m}$, and the undulator spectrum is filtered by the $400 \mu \mathrm{m}$ diamond window. The horizontal size of the aperture corresponds to the projected length of the mirror, $300 \mathrm{~mm} \times \sin \left(0.245^{\circ}\right)$, i.e. full illumination in the meridional direction. The bandwidth of the fundamental ML peak around the working energy (20.25 keV) is considerably wider than the third undulator harmonic, such that most of the heat load in this undulator harmonic is reflected. Note that there is very little overlap between the higher harmonics of the ML and the undulator, such that harmonic contamination of the reflected beam is low. The undulator fundamental, however, falls within the total reflectivity of the multilayer. These lower photon energies therefore have to be filtered out by absorbers or a crystal monochromator.

The reflection of a significant part of the radiation in the central cone reduces the absorbed power around the center of the ML optics (Rebuffi et al., 2020). Due to the variation of the heat load along the length of the ML, for any given set of geometrical parameters (see Fig. 1), locally differing thermal dilatation occurs, leading to residual slope errors due to 'thermal mapping' (see also Section 2 and the example in Section 6.3).

4.1.1. Absorbed radiation calculations. Quantitative calculations of the power maps on the ML surface were calculated for each case in Table 1 using OASYS (Rebuffi \& Sanchez del Rio, 2017) complemented by ad hoc Python scripts. The flux emitted by the undulator is calculated at a screen plane located at the ML position. The flux $\Phi\left(z_{\mathrm{s}}, y_{\mathrm{s}}, E\right)$ [in units of photons $\mathrm{s}^{-1} \mathrm{~mm}^{-2}(0.1 \% \text { bandwidth })^{-1}$ coordinates referring to Fig. 1] is calculated for every spatial coordinate at the screen $\left(z_{\mathrm{s}}, y_{\mathrm{s}}\right)$ and for every photon energy $E$ covering a wide spectrum range. It uses standard undulator radiation flux calculations (e.g. Onuki \& Elleaume, 2003) implemented in the Undulator Radiation application of the $O A S Y S / X O P P Y$ add-on. The energy and space must be sampled with enough resolution in such a way that both (i) the energy integral is similar to the 2D power density (as calculated by the Undulator Power Density application) and (ii) the space integral is similar to the 1D undulator spectrum (as calculated by the Undulator Spectrum application). We used $121 \times 81$ space points covering an area that fully illuminated the ML surface, and $10 \mathrm{eV}$ photon-energy steps, a distance of $d=31 \mathrm{~m}$, and an aperture of $V=1 \mathrm{~mm}$ and $H=L \sin (\theta)$. The angle of incidence, $\theta$, is taken at maximum reflectivity of the corresponding multilayer stripe and calculated in the frame of this work using the 'modified Bragg equation' [equation (9) of Morawe \& Osterhoff (2010)]. The undulator gap was set for maximum flux at the desired photon energy. The spatial distribution of the spectral flux is now expressed as a function of coordinates $(x, y)$ on the ML surface by projecting $(x, y)=$ $\left(z_{\mathrm{s}} \sin \theta, y_{\mathrm{s}}\right)$ the flux $\Phi$ and multiplying it by the pixel ratio $\left(\Delta z_{\mathrm{s}} \Delta y_{\mathrm{s}}\right) /(\Delta x \Delta y)=\sin (\theta)$ to guarantee a constant integrated power,

$$
\Phi_{\mathrm{d}}(x, y, E)=\Phi[x \sin (\theta), y, E] \sin (\theta) .
$$

To obtain the spatial distribution of the power density absorbed by the first ML optics, $p(x, y)$, the spectral flux density for each load case was then post-processed (using Python code) to account for the transmission of the $400 \mu \mathrm{m}$ thick diamond front-end window $T_{\mathrm{FE}}(E)$, and the reflectivity of the multilayer $R_{\mathrm{ML}}(E)$,

$$
p(x, y)=\int_{E}\left[1-R_{\mathrm{ML}}(E)\right] T_{\mathrm{FE}}(E) \Phi_{\mathrm{d}}(x, y, E) \mathrm{d} E .
$$

Multilayer reflectivities $R_{\mathrm{ML}}(E)$ are calculated using the code $I M D$ (Windt, 1998) as an extension of XOP (Sanchez del Rio \& Dejus, 2011).

An example of a resulting absorbed power density map is shown in Fig. 6. We see that the absorbed power density varies significantly across the multilayer.

Separate calculations were carried out for each of the load cases listed in Table 1. For comparison, Fig. 7 shows the absorbed power densities projected onto the meridional direction (length). Only the central part of the mirror (indicated by a solid line in Fig. 7) is illuminated by the central cone and contributes to the reflected beam. 
Table 4

Detailed power calculations of the selected load cases from Table 1.

For each load case we list the ML stripe, grazing angle $\theta$ at maximum reflectivity, stripe bandwidth $\Delta E / E$ and reflectivity $R$. Angles $\theta$ are calculated using the 'modified Bragg equation' [equation (9) of Morawe \& Osterhoff (2010)]. The slit settings $H$ are calculated to overfill a substrate length of $L=300 \mathrm{~mm}$. $P_{\text {in }}$ denotes total incoming power on the ML, $P_{\text {abs, ML1 }}$ absorbed power by first ML and $P_{\text {abs, ML2 }}$ by second, $P_{\mathrm{T}}$ total transmitted power and $P_{\text {abs }}$ total absorbed power through the DMM. Power calculations are performed using $O A S Y S / X O P P Y$ and multilayer reflectivities used $I M D$.

\begin{tabular}{|c|c|c|c|c|c|c|c|c|c|c|c|c|c|c|}
\hline LC & $E(\mathrm{keV})$ & $n$ & Stripe & $\theta\left(^{\circ}\right)$ & $\Delta E / E(\%)$ & $R(\%)$ & $H(\mathrm{~mm})$ & $V(\mathrm{~mm})$ & $P_{\text {in }}(\mathrm{W})$ & $P_{\mathrm{abs}, \mathrm{ML} 1}(\mathrm{~W})$ & $P_{\mathrm{abs}, \mathrm{ML} 2}(\mathrm{~W})$ & $P_{\mathrm{T}}(\mathrm{W})$ & $P_{\text {abs }}(\mathrm{W})$ & $P_{\text {abs }}(\%)$ \\
\hline 1 & 12.0 & 1 & 1 & 0.416 & $12.0 \%$ & $90.6 \%$ & 2.18 & 1 & 642.9 & 497.4 & 36.7 & 108.8 & 534.1 & $83.1 \%$ \\
\hline 2 & 17.0 & 1 & 1 & 0.293 & $12.0 \%$ & $93.0 \%$ & 1.53 & 1 & 255.6 & 120.1 & 23.1 & 112.3 & 143.2 & $56.0 \%$ \\
\hline 3 & 20.3 & 3 & 1 & 0.245 & $12.0 \%$ & $94.6 \%$ & 1.28 & 1 & 513.2 & 401.5 & 22.6 & 89.1 & 424.1 & $82.6 \%$ \\
\hline 5 & 34.0 & 5 & 2 & 0.238 & $6.6 \%$ & $89.8 \%$ & 1.25 & 1 & 495.3 & 377.1 & 37.4 & 80.8 & 414.5 & $83.7 \%$ \\
\hline 6 & 60.0 & 7 & 3 & 0.202 & $2.6 \%$ & $93.8 \%$ & 1.06 & 1 & 393.6 & 277.4 & 34.7 & 81.4 & 312.2 & $79.3 \%$ \\
\hline 7 & 20.3 & & & 0.400 & - & $0.0 \%$ & 2.09 & 1 & 977.8 & 977.8 & - & - & 977.8 & $100.0 \%$ \\
\hline
\end{tabular}

The projected power densities in Fig. 7 range from $0.2 \mathrm{~W} \mathrm{~mm}^{-2}$ to $1.8 \mathrm{~W} \mathrm{~mm}^{-2}$, with a characteristic minimum at the center of the mirror surface. These meridional variations lead to significant residual slope errors due to thermal mapping, see Section 2.

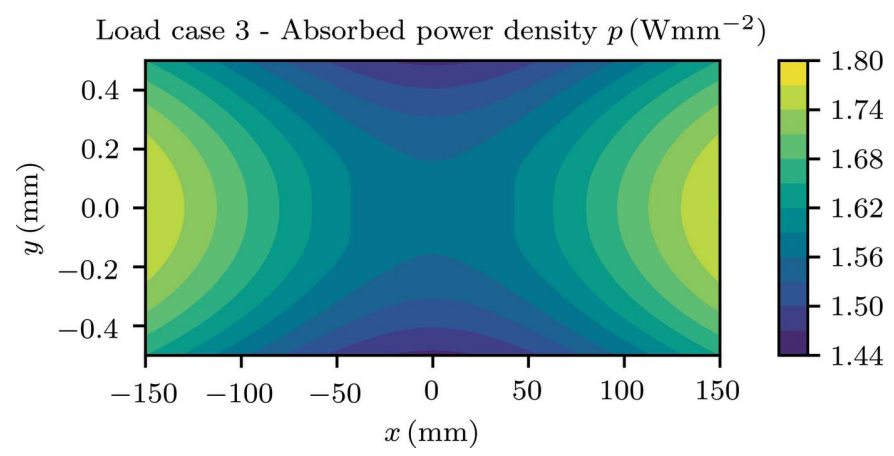

Figure 6

Spatial distribution of the absorbed power by the first DMM mirror for load case 3 at $\theta=0.245^{\circ}$. The undulator spectrum is filtered by the frontend diamond window and projected onto the ML mirror ( $x$ : meridional; $y$ : sagittal), and the power reflected by the ML is taken into account. The covered aperture corresponds to a complete filling of the multilayer substrate length.

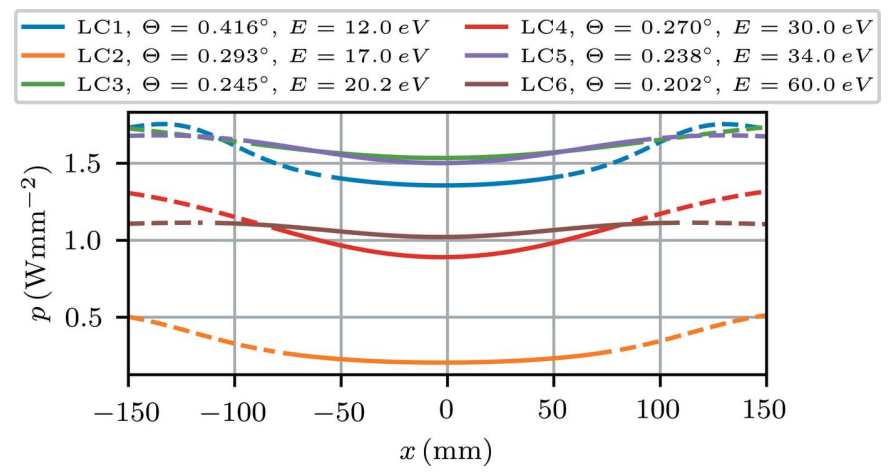

Figure 7

Comparison of the absorbed power densities projected on the multilayer mirror surface for all selected load cases (Table 1) in meridional (length-) direction $x$, averaged over the sagittal direction $(y)$. The solid part of the lines lie in the length covered by 'relevant photons' over which surface errors are evaluated. The general trend of the discussed pit shaped power density profile becomes clear.
Table 4 summarizes the results of the power calculations for all load cases concerning the selected DMM system. The results give information about the incoming power, absorbed power per multilayer optic as well as total transmitted and absorbed power.

\subsection{Surface deformation under thermal load (FEA)}

The typical geometrical parameters (see Fig. 1) are optimized to minimize the bending moment while taking into account the following practical constraints:

(i) The lower limit of $c \simeq 20 \mathrm{~mm}$ is determined by the requirement to keep the maximum substrate temperature below $\sim 100^{\circ} \mathrm{C}$ for all load cases.

(ii) The height and width of the mirror substrate are limited to $d \leq 90 \mathrm{~mm}$ and $b \leq 70 \mathrm{~mm}$ as imposed by the multilayer deposition machine.

(iii) The multilayer stripes should not overlap the smart cut. This, together with the width of the three multilayer stripes $(13 \mathrm{~mm})$, sets the lower limit for the width: $b \geq 2 w_{\text {cut }}+$ $(3 \times 13 \mathrm{~mm})$.

(iv) This leaves the height $h$ and width $w_{\text {cut }}$ of the smart cut as free parameters.

For a given set of geometrical parameters, we use the FEA software ANSYS (http://ansys.com) to calculate the deformation of the multilayer surface under the presented heat loads.

4.2.1. Model. We used the model shown in Fig. 8 to analyze the thermal deformations of the first multilayer of the DMM. For symmetry reasons of the heat load in the meridional direction (compare Fig. 7) only half of the mirror is modeled for the thermal-mechanical analysis. The multilayer stripes are arranged in such a way that the central laying stripe covers the low energies $12 \mathrm{keV}$ to $24 \mathrm{keV}$, with the remaining stripes on each side laterally off-centered by $13 \mathrm{~mm}$.

The mesh used for the calculation is a sweep mesh in the mirror length $(x)$, starting from the front face. In the sagittal direction $(y)$ the element size varies from $0.17 \mathrm{~mm}$ in the center of the beam footprint to $2 \mathrm{~mm}$ at the edge, whereas in the meridional direction $(x)$ it is constant at $2 \mathrm{~mm}$. The mesh used was validated with a convergence study revealing low influence of a finer result on the relevant results (slope errors). In the sagittal direction, the footprint width is $1 \mathrm{~mm}$ and six 


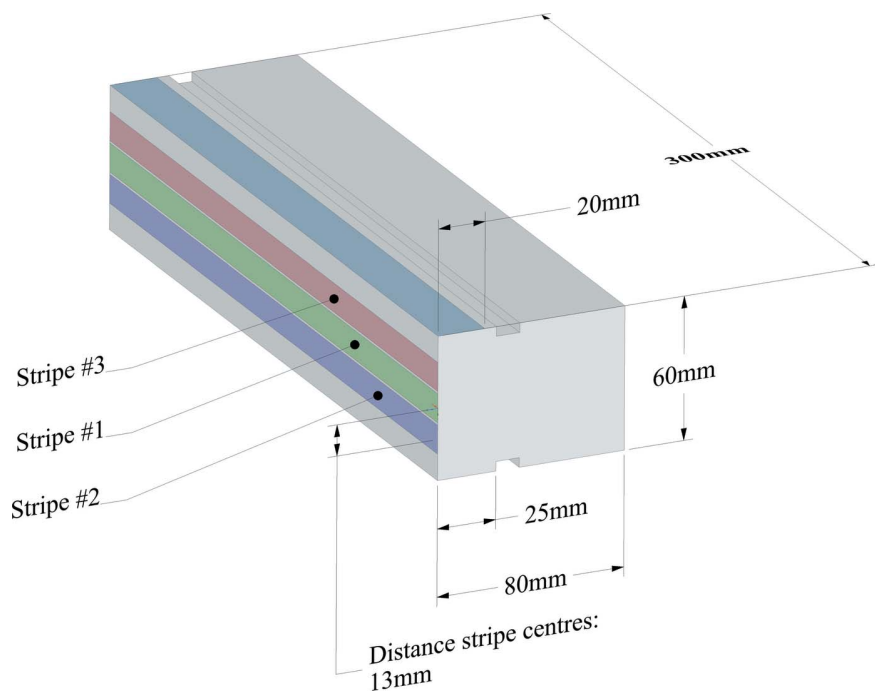

Figure 8

Calculated model geometry. This geometry resulted from various different tried geometrical parameter combinations (compare Fig. 1) as the most promising configuration. The three stripes are each $13 \mathrm{~mm}$ wide, stripe 1 in the central position and both 2 and 3 in lateral positions with $1 \mathrm{~mm}$ distance between each coating. The mirror is cooled over the height marked in light blue $(20 \mathrm{~mm})$.

elements over the width are used for the sagittal calculations (thus 11 nodes over width).

4.2.2. Cooling conditions. The mirror cooling is modeled by means of a thermal convection coefficient of $3 \mathrm{~kW} \mathrm{~m}{ }^{-2} \mathrm{~K}^{-1}$ applied on the top sides of the mirror. This is a typical value for indirect cooling with clamped coolers which was confirmed by measurements and used in similar analysis (Zhang et al., 2003; Chumakov et al., 2014; Brumund et al., 2021). The cooling liquid is water at room temperature $\left(22^{\circ} \mathrm{C}\right)$.

4.2.3. Absorbtion modeling. The heat-load effects were analyzed in a simplified model. The depth-distributed absorption of X-rays in the multilayer was approximated by surface deposition of the heat load. This is possible due to the comparably small grazing angle and hence absorption close to surface. This becomes particularly clear in the comparison with single-crystal monochromators, where this approximation can be made already in cases with higher incidents angles (Zhang et al., 2003; Brumund et al., 2021).

4.2.4. Material data used. Non-linear Si material properties were used for the investigated model, similar to the ones presented in detail by Brumund et al. (2021). Thermal conductivity values are from Touloukian et al. (1971) whereas thermal dilatation coefficients are from measurements taken by Middelmann et al. (2015). Anisotropic elastic mechanical properties are taken for $\mathrm{Si}(100)$ (Zhang et al., 2014). The use of another orientation can lead to a reduction of thermal slopes of up to $8.9 \%$ for $\mathrm{Si}(111)$ as the same source states.

\subsection{Extraction of curvatures and residual slope errors from the FEA results}

From the FEA calculations we obtain height profiles $w(x, y)$ of the reflecting surface for the selected load cases, where $w>0$ indicates an inward displacement $(z>0, c f$. Fig. 1$)$. The partial derivatives of the surface profiles with respect to the relevant coordinate then give the local slopes in the meridional and sagittal directions.

4.3.1. Meridional direction. For the meridional direction we extract the height profile $w\left(x, y_{0}\right)$ at the center $y_{0}$ of the relevant stripe. The slopes $s_{\mathrm{m}}(x)$ are calculated as the derivative $s_{\mathrm{m}}(x)=\partial w\left(x, y_{0}\right) / \partial x$. The r.m.s. slope error $\sigma_{\mathrm{m}}$ is the standard deviation of $s_{\mathrm{m}}(x)$.

For the curvature calculation the slope is fitted with a straight line, $s_{\mathrm{fit}}=\kappa_{\mathrm{m}} x+c_{\mathrm{m}}$. Here $c_{\mathrm{m}}$ is a constant angular offset that can be corrected by aligning the mirror. In our calculations, however, the meridional slope at $x=0$ is always zero, $c_{\mathrm{m}}=0$, due to symmetry. The linear coefficient $\kappa_{\mathrm{m}}$ gives the curvature of the circle with radius $R_{\mathrm{m}}=1 / \kappa_{\mathrm{m}}$ that, after subtraction from the height profile, minimizes the slope error. The residual slope error is then $\Delta \alpha_{\mathrm{m}}(x)=s_{\mathrm{m}}\left(x, y_{0}\right)-\kappa_{\mathrm{m}} x-$ $c_{\mathrm{m}}$, and the r.m.s. residual slope error $\Delta \alpha_{\mathrm{m}}$ is the standard variation thereof.

The slope errors are relevant only within an assumed 'central cone' (i.e. the area where the reflected beam has a significant intensity, see Brumund et al., 2021), therefore the meridional r.m.s. slope errors $\Delta \alpha_{\mathrm{m}}$ and $\kappa_{\mathrm{m}}$ were evaluated only over the length $L_{\text {eval }}=H_{\text {eval }} / \sin (\theta)$, where $H_{\text {eval }}=0.8 \mathrm{~mm}$ is the approximate width (horizontal size) of the undulator's central cone at the position of the DMM. This effect is more pronounced at the ESRF-EBS than ESRF-1, as the smaller horizontal emittance leads to a smaller central cone ${ }^{5}$. The value $H_{\text {eval }}$ is assumed constant for all load cases.

4.3.2. Sagittal direction. The sagittal profile is $s_{\mathrm{s}}(y)=$ $\partial w(0, y) / \partial y$, from where the r.m.s. slope error $\sigma_{\mathrm{s}}$ is obtained. Residual slope errors $\Delta \alpha_{\mathrm{s}}$ are calculated after subtracting the slope of the sagittal circle $s_{\text {fit }}=\kappa_{\mathrm{s}}\left(y-y_{0}\right)+c_{\mathrm{s}}$ with curvature $\kappa_{\mathrm{s}}$ that minimizes the r.m.s. slope error. In the sagittal direction we have a slope offset when $y_{0} \neq 0$, i.e. $c_{\mathrm{s}} \neq 0$, due to the nonsymmetrical heat load situation. Sagittal errors are evaluated at the center of the relevant ML stripe over the whole beam footprint height $(V=1 \mathrm{~mm})$, that is between $y=y_{0} \pm 0.5 \mathrm{~mm}$.

\section{Results}

We now turn to the discussion of how the geometrical parameters affect the curvature and residual slope errors of the mirrors. First, we present qualitatively how geometrical parameters influence the meridional slope errors (Section 5.1). Then we turn towards the results of the presented DMM design, first in the meridional (Section 5.2) and then in the sagittal direction (Section 5.3).

\subsection{Qualitative effect of varying the geometrical parameters}

This section demonstrates how the typical geometrical mirror parameters as defined by Khounsary (1999) influence

\footnotetext{
${ }^{5}$ Potentially, one could vary the multilayer's $d$-spacing along the meridional direction to 'track' the undulator's emission spectrum and thus reduce the variations of the power density. Most likely, however, this would work for only one combination of undulator setting and grazing angle $\theta$. Here, we therefore consider only the case of constant $d$-spacings.
} 


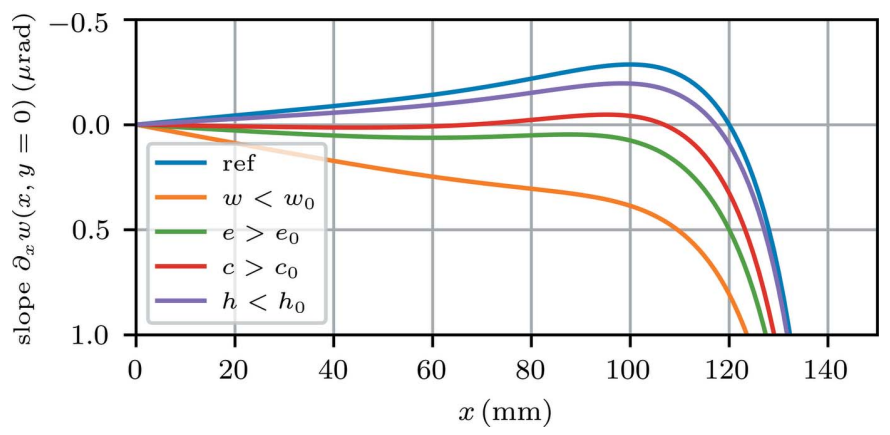

Figure 9

Qualitative demonstration of the influence of typical parameters on the X-ray mirror thermally induced slopes along the optics length $(x)$. Downwards curved lines $\left(\partial_{x} w>0\right)$ correspond to convex shape. The plot shows the influence of geometrical parameters presented in Fig. 1 on surface slopes at constant beam power along the mirror length.

the optical surface distortion. The reference parameters used for the simulations are similar to the ones in the main work for this project and described in Section 4.2.

Figure 9 shows how a modification in some of the geometrical parameters stated in Fig. 1 affects the surface shape, aiming to reduce thermal bending effects. Usually the optic's width $b$, length $L$ and beam size are defined by the user. The height $d$ is often determined by the need of sufficient space for mechanical clamping. The main effects, sorted by significance, are:

(i) $w_{\text {cut }}$. Notches 'confine' the colder area, as Khounsary (1999) pointed out: if $w_{\text {cut }}$ is decreased $\left(w_{\text {cut }}<w_{0}\right)$, the bottom part of the mirror below the neutral axis is further cooled, thus reducing its resistance to the hot beam footprint component of the bending moment. The result is a convex bending. Similarly, if $w_{\text {cut }}>w_{0}$, the cold zone is more confined, cooling less the bottom part of the optics, resulting in a concave bending.

(ii) $e$ and $c$. Side height $e$ and cooled height $c$ both also influence the amount the bottom section is cooled. Increasing these parameters cools the area below the neutral axis, resulting in a convex shape.

(iii) $h$. The notch height adds or removes some material of the bottom bulk above the neutral axis. Usually this material is 'warm' with respect to the thermal bending and hence induces a 'downbending'. If more 'warm' material is added $\left(h<h_{0}\right)$, the shape becomes more convex; if material is removed $(h>$ $h_{0}$ ), it becomes more concave. However, this influence is the smallest of the shown ones and one could select a height that is reasonably well to machine.

\subsection{Results - meridional}

To find good candidates of geometrical parameter sets, we first studied the influence of the geometrical parameters from Fig. 1 on the thermal bending and the residual slope errors, using load case 3 and a fixed notch height $h=10 \mathrm{~mm}$. The results for different geometry combinations are presented in Fig. 10. We note the following trends (see also Section 5.1):

(i) The width $b$ of the mirror has little influence on the bending radius $R_{\mathrm{m}}=1 \kappa_{\mathrm{m}}$, because the temperature integral along $y, I T(x, z)$ [equation (2)], does not change much. Increasing the width (orange curve) decreases the cooling efficiency and thus increases the temperature field above the notch (close to footprint) more than below, leading to a slightly more convex shape.

(ii) Increasing the height $d$ of the substrate (green curve) stiffens the mirror against thermal bending, thus reducing the influence of the cut depth $w_{\text {cut }}$.

(iii) Increasing the cooling surface $c$ leads to a more convex shape and reduces the residual slope errors (red curve). The more convex shape is due to increased cooling of the volume below the notch and the resulting negative bending moment (cf. Section 5.1). Better cooling also reduces the global temperature variations and thermal gradients, lowering the residual slope errors. Both effects are further enhanced by non-linear material properties, in particular higher thermal conductivity at lower temperatures.

(iv) Increasing $w_{\text {cut }}$ leads generally to a more convex shape, but has little influence on the residual slope errors [Fig. 10(b)], as $w_{\text {cut }}$ mostly affects the temperature below the notch.

(v) Edge effects due to longitudinal expansion of the unconstrained upstream and downstream faces of the substrates generate slope errors at the extremities. The length $L$ of the mirror should therefore be longer than the intended active length.

The depth of the smart cut, $w_{\text {cut }}$, and cooled height, $c$, thus have the strongest effect on the meridional bending moment.
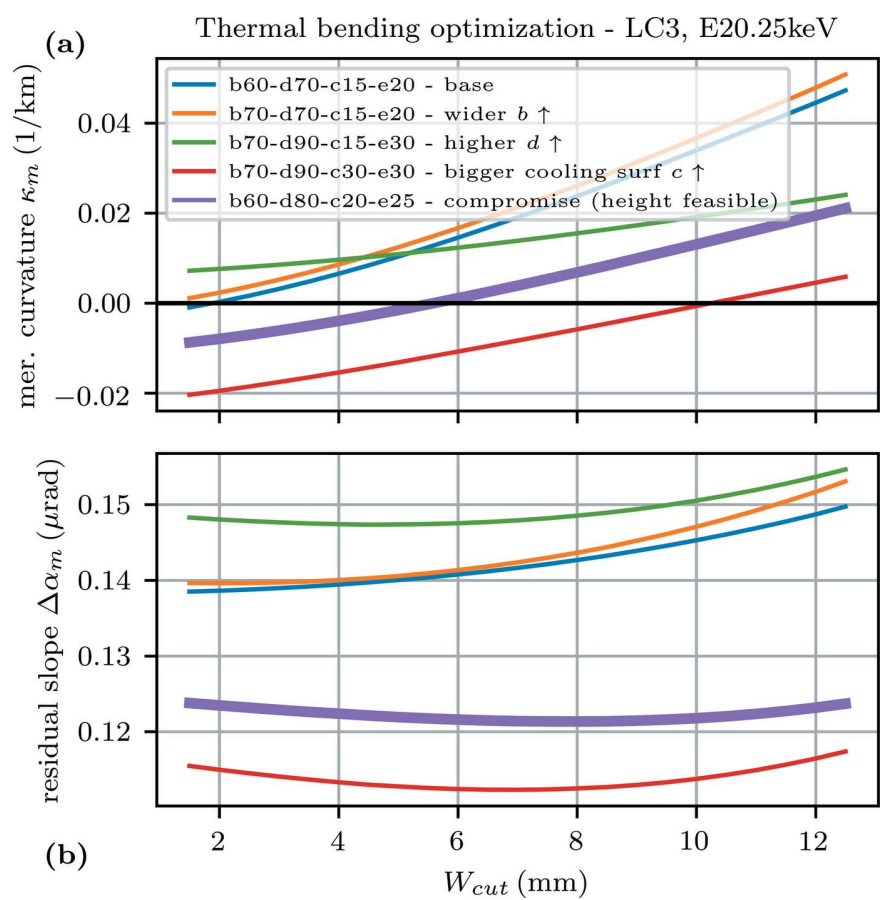

Figure 10

Parameter study results of the influence of the presented main crosssection parameters in Fig. 1 for the fixed load case $3(E=20.25 \mathrm{keV})$. The legend code states the selected geometry configuration, all dimensions being in mm. (a) Main curvature in the meridional direction $\kappa_{\mathrm{m}}=1 / R_{\mathrm{m}}$ (curvatures $\kappa_{\mathrm{m}}>0$ meaning convex shape) and (b) residual r.m.s. slope errors $\Delta \alpha_{\mathrm{m}}$ in the meridional direction (after subtracting the main curvature). 
In particular, increasing $c$ can counteract the convex shape induced by the pit-shaped power profiles (compare Fig. 12).

From this initial general parameter study we selected a promising candidate for which all load cases were considered, with label b60-d80-c20-e25 (referring to the main dimensions in Fig. 1). The violet lines in Fig. 10 show the r.m.s. slope errors $\sigma_{\mathrm{m}}$ and the main curvature $\kappa_{\mathrm{m}}=1 / R_{\mathrm{m}}$.

Due to the symmetry of the heat load about the center of the mirror, $x=0$, there is no constant slope offset $c_{\mathrm{m}}=0$ ( $c f$. Section 4.3). For stripe 1 we used $y_{0}=0$, but for stripes 2 and 3 the integrals are evaluated at $y_{0}= \pm 13 \mathrm{~mm}$.

We calculated the response of the pre-selected geometry b60-d80-c20-e25 for all load cases and study the variation of the smart cut parameters, in particular $w_{\text {cut }}$ (Fig. 11). The following trends are observed:

(i) Some load cases (LC 2 and 4) have a strong tendency to bend the mirror into a concave shape $\left(\kappa_{\mathrm{m}}>0\right)$, whereas others (LC 6, 3 and 5) for some values of $w_{\text {cut }}$ are rather leading to a convex shape [see Fig. 11(b)].

This makes it difficult to optimize the thermal bending for several different load conditions at the same time.

(ii) Therefore there is always at least one load case (here LC 4), where the r.m.s. slope error $\sigma_{\mathrm{m}}$ is above $\sim 1 \mu \mathrm{rad}$ [Fig. 11(a)].

(iii) The radius of curvature induced by thermal bending is on the order of $\kappa_{\mathrm{m}} \simeq 0.02 \mathrm{~km}^{-1}$, thus $R_{\mathrm{m}} \simeq 50 \mathrm{~km}$.

(iv) The geometrical parameters have little to no effect on the residual slope $\Delta \alpha_{\mathrm{m}}$ errors due to thermal mapping and end effects [Fig. 11(c)].

(v) The residual slope errors are below $\sim 0.25 \mu \mathrm{rad}$ for all load cases.
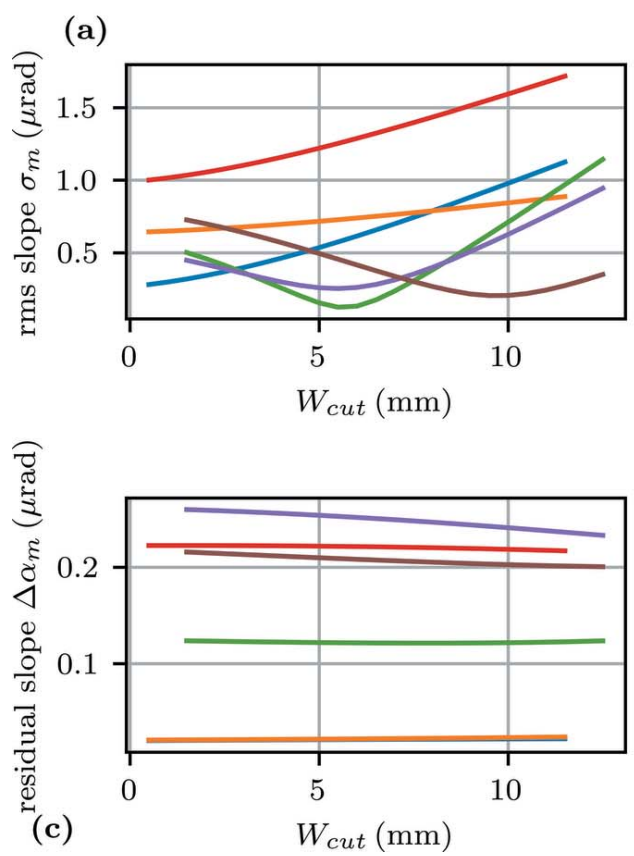

For the selected mirror configuration, the maximum substrate temperature under the worst-case heat load (LC7) is about $98^{\circ} \mathrm{C}$.

\subsection{Results - sagittal}

An analysis similar to the one presented in the previous section was carried out for the sagittal direction. Whereas in the meridional direction the beam completely illuminates the substrate (overfilling), in the sagittal (vertical) direction the size of the beam spot is significantly smaller than the width of the mirror substrate. This leads to results that are qualitatively different from those of the meridional direction presented in the previous section.

For the load cases using the off-center ML stripes, the slope offset $c_{\mathrm{s}}$ is not zero ( $c f$. Section 4.3).

For the sagittal height profiles, we find:

(i) The shape in the sagittal direction is always convex, as the height of the beam spot $V \leq 1 \mathrm{~mm}$ is always smaller than the width of the substrate $b=60 \mathrm{~mm}$.

(ii) Because the footprint is much smaller than the width of the mirror, there is no significant bending over the whole width of the substrate - local thermal mapping is dominant. However, when considering the deformation within the beam spot, there is a non-negligible curvature $\kappa_{\mathrm{s}}$ and significant residual slope errors $\Delta \alpha_{\mathrm{s}}$ due to edge effects.

(iii) Even when taking the 'forgiveness factor' into account, this curvature leads to non-negligible defocusing of the beam, as we discuss below.

(iv) For beam spots away from the center axis (LC 4, 5 and 6 , which use stripes 2 and 3 ) there is also a constant offset $c_{\mathrm{s}}$ to
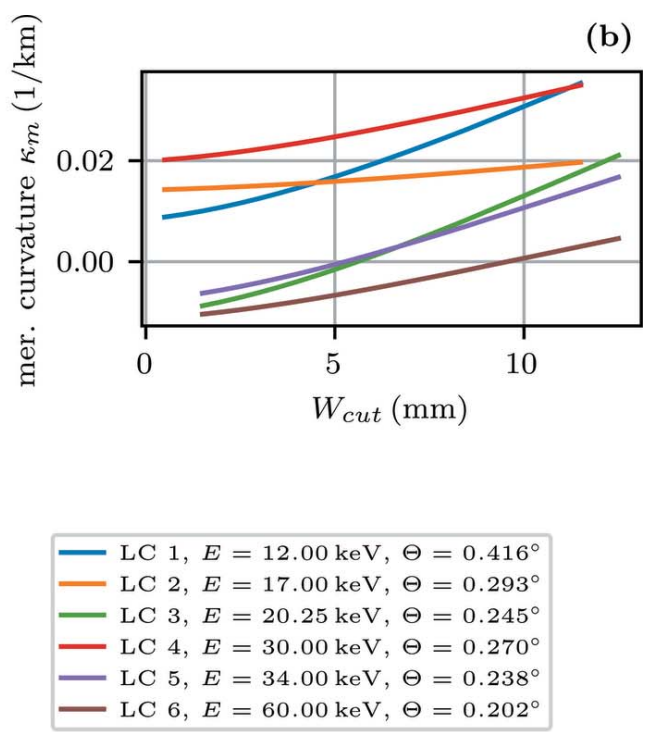

Figure 11

Results of the meridional slope error optimization for selected load cases. The thermal bending moment was minimized by geometrical modification of the mirror cross section. Slope errors $\sigma_{\mathrm{m}}(a)$, curvature $\kappa_{\mathrm{m}}(b)$ and residual slope errors $\Delta \alpha_{\mathrm{m}}(c)$ are plotted as a function of the geometrical parameter $w_{\text {cut }}$ (see Fig. 1) for the pre-selected configuration b60-d80-c20-e25 (mirror width $b=60 \mathrm{~mm}$, height $d=80 \mathrm{~mm}$, cooled and side height $c=25 \mathrm{~mm}$ and $e=$ $30 \mathrm{~mm}$, smart-cut height $h=10 \mathrm{~mm}$ ). 
Table 5

Numerical results from the multilayer geometry optimization for the selected geometry configuration b60-d80-c20-e 25 and $w_{\text {cut }}=4 \mathrm{~mm}$ notch depth.

The table shows, for both the meridional and sagittal plane: the r.m.s. slope error $\sigma_{i}=$ r.m.s. $\left(\mathrm{d} w / \mathrm{d} x_{i}\right)$ before fit of a sphere with radius $R_{i}$ that minimizes the residual slope errors $\Delta \alpha_{i}$ (r.m.s.). The equivalent focal length of the best sphere fit is also stated $\left(f_{i}\right)$.

\begin{tabular}{|c|c|c|c|c|c|c|c|c|c|c|}
\hline \multirow[b]{2}{*}{$\mathrm{LC}$} & \multirow[b]{2}{*}{$E(\mathrm{keV})$} & \multirow[b]{2}{*}{$\theta\left({ }^{\circ}\right)$} & \multicolumn{4}{|l|}{ Meridional } & \multicolumn{4}{|l|}{ Sagittal } \\
\hline & & & $\sigma_{\mathrm{m}}(\mu \mathrm{rad})$ & $R_{\mathrm{m}}(\mathrm{km})$ & $f_{\mathrm{m}}(\mathrm{m})$ & $\Delta \alpha_{\mathrm{m}}(\mu \mathrm{rad})$ & $\sigma_{\mathrm{s}}(\mu \mathrm{rad})$ & $R_{\mathrm{s}}(\mathrm{m})$ & $f_{\mathrm{s}}(\mathrm{m})$ & $\Delta \alpha_{\mathrm{s}}(\mu \mathrm{rad})$ \\
\hline 1 & 12.00 & 0.416 & 0.462 & 68.7 & 250 & 0.021 & 10.690 & -28.3 & -1950 & 0.144 \\
\hline 4 & 30.00 & 0.270 & 1.158 & 42.7 & 101 & 0.222 & 6.972 & -45.8 & -4859 & 0.081 \\
\hline 5 & 34.00 & 0.238 & 0.298 & -405.3 & -842 & 0.256 & 12.349 & -25.6 & -3079 & 0.115 \\
\hline 6 & 60.00 & 0.202 & 0.570 & -126.5 & -223 & 0.212 & 7.828 & -40.4 & -5726 & 0.064 \\
\hline
\end{tabular}

the slope. However, the optical effect of this offset is negligible, due to the 'forgiveness factor'.

Our simulations show very little influence of the geometrical parameters on the sagittal slope errors and sagittal main curvature, since these are mainly due to local dilatation in the beam footprint. This cannot be influenced significantly by changing the geometry of the substrate. Only lowering the locally acting heat load (i.e. smaller grazing angle) could have significant effects.

\section{Discussion}

The simulations presented here contain of course some simplifications. Firstly, we calculated only the thermal deformation of the first ML optic of the DMM system. The deformations of the second ML were ignored, as the heat load absorbed by the second ML is more than ten times smaller (compare Table 4) and thus slope errors drastically reduced. However, the calculation of this second ML in future calculations remains a possible additional task.

Furthermore, for the thermal analysis we assumed that the cooling coefficient is constant over the mirror's length. This simplification corresponds to a constant clamping pressure of the cooler to the cooled mirror side, which is obviously an idealized situation. We expect that irregularities of the clamping pressure will also translate into additional residual thermal slopes to induced additional thermal mapping effects from non-uniform cooling.

Lastly, the heat load was considered symmetric in the longitudinal direction about the $x=0$ plane. This assumed a perfectly aligned optic.

\subsection{Slope errors, main curvatures and induced focal lengths}

Table 5 summarizes the results of the optimization including, for meridional and sagittal planes, the slope errors, fitted radii and residual slope errors after best toroidal fit. We first analyze the slope errors $\sigma_{\mathrm{m}, \mathrm{s}}$ before subtracting the best toroidal fit, then the focusing effect of the main curvature radii $R_{\mathrm{m}, \mathrm{s}}$ and their induced focal lengths $f_{\mathrm{m}, \mathrm{s}}$. Lastly, we discuss the residual slope errors $\Delta \alpha_{\mathrm{m}, \mathrm{s}}$ after removing the main curvature and their effect.

As shown in Table 5, sagittal slope errors before best fit (up to $\sim 12.3 \mu \mathrm{rad}$ ) are more than one magnitude larger than the meridional slope errors (up to $\sim 1.2 \mu \mathrm{rad}$ ). These errors are mainly due to a curvature that is much larger in the sagittal direction ( $R_{\mathrm{s}}$ from $\sim 25$ to $220 \mathrm{~m}$ ) than in the meridional plane ( $R_{\mathrm{t}}$ from $\sim 42$ to $405 \mathrm{~km}$ ). This thermally induced bending curvature produces a parasitic focusing of the beam, usually undesired, that is better understood using the effective focal lengths given by the Coddington equations (Kingslake, 1994; Willmott, 2019),

$$
f_{\mathrm{m}}=\frac{1}{2} \sin (\theta) R_{\mathrm{m}}
$$

$$
f_{\mathrm{s}}=\frac{1}{2} \frac{1}{\sin (\theta)} R_{\mathrm{s}},
$$

for the meridional and sagittal cases, respectively. In the sagittal direction, $f_{\mathrm{s}}$ is larger than $2 \mathrm{~km}$ (with negative or convex curvature, thus defocusing), whereas in the meridional plane the load case 4 leads to the smallest focal length of $101 \mathrm{~m}$ (focusing). When comparing these focal lengths with the typical length of a synchrotron beamline (30 to $200 \mathrm{~m}$ ) we see that the sagittal defocusing effect is negligible, whereas the meridional (de)focusing effect must be taken into account when designing the beamline optics. Furthermore, this shows that, for multilayer mirrors with small grazing angle, one should prioritize minimizing the meridional curvature in the thermal design.

Different meridional and sagittal induced focal lengths leads to an astigmatism. This requires astigmatic optics (e.g. 1D refractive lenses) to fully correct these introduced systematic distortions.

\subsection{Residual slope errors}

Our simulations show that the residual slope errors depend only weakly on the geometrical parameters of the mirror, and in particular on $w_{\text {cut }}$ [Figs. 10(b) and 11(c)]. The origin is the 'thermal mapping' that results from local differential thermal dilatation induced from heat-load variations over the optics surface. To some extent these errors are also provoked by the end deformation effects due to the fact that the substrate is of finite length.

However, we find a pronounced correlation between the residual meridional slope errors [see Fig. 11(c)] and the following parameters: 
(i) The grazing angles, which differ from one load case to another.

(ii) The lateral position of the illuminated stripe.

(iii) Variation of heat load over the evaluated footprint area.

Load cases 3-6 use the smallest grazing angles, thus the lengths over which the slope errors are evaluated are the biggest (solid lines in Fig. 7). Consequently, the evaluation of these load cases includes more of the strong end effects (compare Fig. 9) which increase residual slope errors.

Load cases 4-6 all utilize an off-center stripe, leading to slightly higher residual slope errors in comparable conditions (see Section 6.3). Load case 3 is comparable with load case 5 in terms of evaluated footprint length (about $L_{\text {rel }} \simeq 198 \mathrm{~mm}$ ) and heat load (see Fig. 7). However, load case 3 lays on the central stripe, which leads to smaller residual slope errors $\Delta \alpha_{\mathrm{m}}=$ $122 \mathrm{nrad}$ by a factor of more than two if compared with case 5 . In the example of Fig. 12 the heat load on the lateral position leads to an increased curvature of the slope in this plot, also indicating higher residual errors after best spherical fit.

Residual slope errors after best fit are of similar order in both planes. Meridional residual slope errors after removal of best spherical fit, thus incorrigible errors, reach up to $0.26 \mu \mathrm{rad}$. Due to the 'forgiveness factor' and the small grazing angles $\theta_{\mathrm{B}}$, however, the influence of residual sagittal slope errors $\Delta \alpha_{\mathrm{s}}$ on the exit beam distortions in the sagittal plane $\Delta \theta_{\mathrm{s}}$ are decreased by a factor

$$
\frac{\Delta \theta_{\mathrm{s}}}{\Delta \theta_{\mathrm{m}}}<\sin \left(\theta_{\mathrm{B}}=0.4^{\circ}\right)=7 \times 10^{-3},
$$

and assuming similar residual slope errors $\Delta \alpha_{\mathrm{m}}=\Delta \alpha_{\mathrm{s}}$. This effect was observed earlier and recently investigated on monochromators at far higher grazing angles (DiGennaro et al., 1988; Brumund et al., 2021). Resulting beam distortions in the sagittal plane $\Delta \theta_{\mathrm{s}}$ caused by residual sagittal slope errors

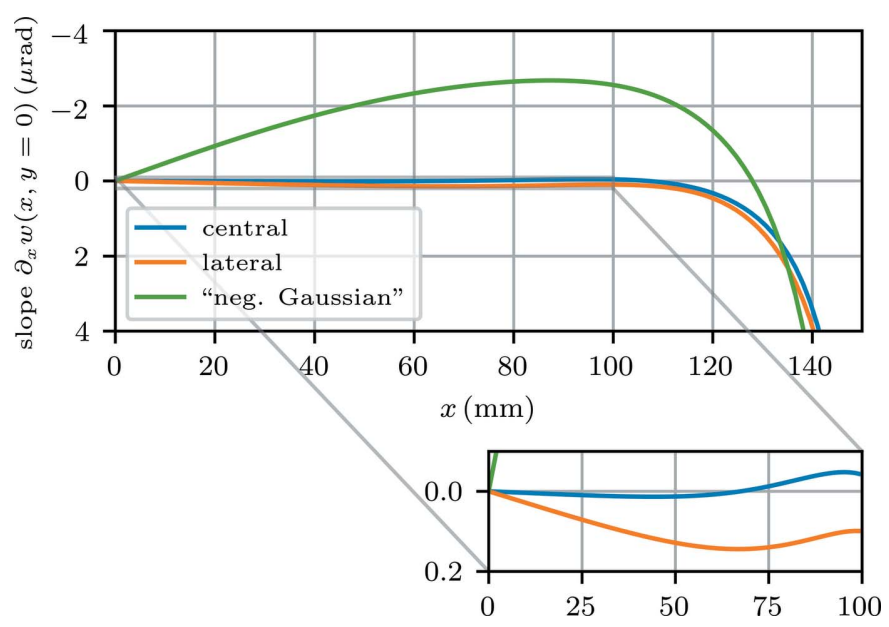

Figure 12

Influence of typical parameters on X-ray mirror thermally induced slopes along the optics length $(x)$. Downwards curved lines $\left(\partial_{x} w>0\right)$ correspond to convex shape. The plot shows the influence of different power profiles on mirror deformations: constant power along the optic's length on the central position, on the lateral position and 'negative' Gaussian power profile with pit on the center of the mirror.
$\Delta \alpha_{\mathrm{s}} \simeq 0.13 \mu \mathrm{rad}$ are thus decreased to the level of a nanoradian, making their influence irrelevant for further analysis.

Predicted thermal residual slope errors of the optimized DMM system (grazing angles $0.2-0.4^{\circ}$ ) are up to $\sim 250 \mathrm{nrad}$ (r.m.s.) in magnitude (for the $34 \mathrm{keV}$ load case). This is significantly bigger than polishing errors (50-160 nrad) and slope errors from mechanical clamping (50-100 nrad), thus confirming that the thermal optimization is necessary.

For the main working energy $(17 \mathrm{keV})$ the expected residual thermal slope errors of $20 \mathrm{nrad}$ are much smaller, however. This should make their optical non-correctable effects almost irrelevant for this case. For this case, possibly only the introduced equivalent focal length of about $160 \mathrm{~m}$ from thermal bending will have an impact on the optical performance. This effect should be correctable with downstream 1D lenses, if necessary.

In future studies, it could be of interest to further investigate the residual height errors in view of correcting them with more advanced optics. This, however, is beyond the scope of this work.

\subsection{Influence of power profiles}

The influence of typical absorbed beam power profiles is illustrated in Fig. 12. This is only for demonstration purposes and exact numerical results as well as numerical values are not explained further here. The same counts for details of the calculation method, which is similar to that in the main work for this project and described in Section 4.2.

A constant beam power $p$ on a lateral position (orange curve) usually leads to a slightly more convex bending of the optics. In addition, residual slope errors after removing the best spherical fit are typically slightly higher for the power load on the lateral position. This depends, however, on the considered length for this fit.

The effect of a non-uniform power profile is shown by the 'neg. Gaussian' curve (green), where the power profile is the sum of a constant power and a negative Gaussian distribution $g(x): p(x)=p-g(x) \cdot g(x)$ is chosen with a standard deviation shorter than the optics length $\sigma<L$ and an amplitude three times smaller than $p$. This can approximate the power profile on a multilayer optics, with a significant part of the central cone power being reflected and not absorbed.

It can be seen that the varying heat load significantly alters the thermal distortions, with the height profile approximately following the power profile. This is the thermal 'mapping' effect due to local thermal dilatation.

The slope errors caused by thermal mapping cannot be controlled with the 'smart cuts' and thus increase the residual slope errors. As Khounsary \& Yun (1996) suggested, the best method to minimize these errors is to keep the absorbed heat load along the X-ray optics as constant as possible. This was feasible for third-generation sources with horizontally deflecting total reflection mirrors, as the heat load in the horizontal direction was relatively constant. For multilayer optics under fourth-generation source beams like at the ESRF-EBS, where the undulator harmonics have smaller 
bandwidth than the ML, this condition cannot be met, and therefore a significant part of the incident radiation is reflected. Then the absorbed heat load varies significantly over the optics surface, as recently shown (Rebuffi et al., 2020).

6.3.1. Error from wrong heat load. Figure 13 demonstrates the importance of taking into account the reflected beam power when predicting the thermal deformations and residual slope errors on a practical example of one of the selected load cases (LC 3). We first assumed that there is no reflected beam power and optimized the multilayer geometry for the 'direct' undulator heat load (blue) or a heat load that is constant along the length of the substrate (orange). In these cases the calculated residual slope errors are $\sim 40 \mathrm{nrad}$, i.e. significantly smaller than the $\sim 120$ nrad when the reflected heat load is taken into account (red). This red curve corresponds to the way we considered the heat load more realistically in the frame of this paper.

If, however, the mirror is optimized neglecting the reflected beam power, the difference in assumed and real heat load leads to much larger slope errors (green). In particular, the substrate shows a strong concave curvature due to thermal bending, similarly as shown by the 'neg. Gaussian' case in Fig. 12. The blue line shows the optimized case for the 'direct' undulator heat load without consideration of the multilayer reflectivity with minimum main curvature. If the more realistic heat load which considers reflectivity is applied to this geometry (smart cut $w_{\text {cut }}=12 \mathrm{~mm}$ ), the resulting slope errors (green) now contain a main curvature of about $52 \mathrm{~km}$. This corresponds to an effective focal length of $104 \mathrm{~m}\left(\theta=0.23^{\circ}\right)$ resulting from erroneous assumption of the wrong heat load.

Again, due to the strong variations in the absorbed heat load of the chosen load cases, it is impossible to simultaneously optimize the geometry for all load cases. This becomes clear looking at the strongly varying results in Table 5 and Figs. 11(a) and 11(b). Thus, the chosen final configuration is

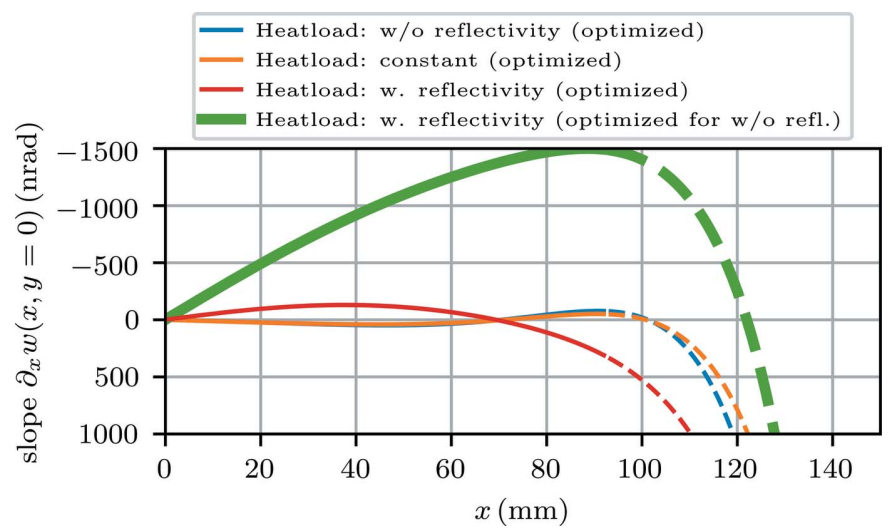

Figure 13

Significance of the use of the correct heat load to predict multilayer thermal deformations. Different heat loads were applied on the basis of load case 3 in $d=30 \mathrm{~m}$ from the source: direct heat load without consideration of ML reflectivity (blue), constant heat load $p=$ $1.5 \mathrm{~W} \mathrm{~mm}^{-2}$ (orange) and the previously presented heat load considering the ML reflectivity (red, $c f$. Fig. 7). The green line represents the error made if the ML is optimized for the given load case and heat load without consideration of ML reflectivity. always a compromise in favor of a small number of load cases in terms of minimized main curvature.

\section{Summary and conclusions}

We investigate the thermal deformation of a multilayer mirror under the heat load of the white beam of an undulator at the ESRF-EBS. Due to selective absorption by the substrate of the radiation from different undulator harmonics, the heat load is not uniform or of simple Gaussian shape across the mirror surface, as assumed in previous studies. A significant part of the white beam near the beam axis is reflected, and therefore does not contribute to the heat load. This creates a 'hollow' heat-load profile, with a local minimum in the center of the mirror. The bending moment is therefore not constant along the length of the mirror, and the mirror would manifest non-negligible concave curvature if its geometry was optimized following the previous methods (i.e. using constant or Gaussian heat load). The optimization of the geometrical parameters of the multilayer mirror for a DMM presented here takes into account this variation of the heat load along the mirror, resulting in more accurate geometrical parameters, in particular the depth of the 'smart cut'.

Furthermore, we study the deformation height and slope profiles separating the effect of 'systematic' slope errors (corresponding to a constant radius of curvature) and residual slope errors (attributed to 'thermal mapping'). Systematic slope errors lead to an effective (de-)focusing of the beam. This can be corrected by adapting up- or downstream focusing optics. Due to the grazing angle of incidence, the footprint of the beam is highly asymmetric. This leads to very different radii of curvature in the sagittal and meridional directions. The difference is only partially compensated by the 'forgiveness factor' in the Coddington equations (Kingslake, 1994; Willmott, 2019), such that the effective focal lengths in the meridional and sagittal directions are different. The correcting optics must therefore be astigmatic.

Residual slope errors, on the other hand, are much more difficult to correct. When adjustable focusing optics are available, the design of the multilayer mirror and beamline optics should therefore aim to minimize the residual slope errors.

For the DMM system for the planned new EBSL beamline ID03, the predicted meridional residual slope errors reach up to $\sim 250 \mathrm{nrad}$ (r.m.s., for load case 5, $34 \mathrm{keV}$ ). How these slope errors affect the experiment depends on the downstream optics configuration - which we do not discuss here. For the main working energy $(17 \mathrm{keV})$ the expected residual thermal slope errors of 20 nrad are much smaller. The smallest equivalent focal length of $100 \mathrm{~m}$ due to thermal deformation is predicted for the optimized mirror for the $30 \mathrm{keV}$ working energy.

The largest residual sagittal slope error $\left(\Delta \alpha_{\mathrm{s}}=144 \mathrm{nrad}\right)$ is found for load case 1 (12 keV). The 'foregiveness factor' $\sin (\theta)$ reduces the resulting beam deviations to 1 nrad r.m.s., which is negligible. 
In future projects, the residual slope errors could potentially be reduced by one or more of the following means:

(i) Using another substrate material with lower thermal dilatation coefficient [or by using Si at cryogenic temperatures (Cutler et al., 2020)].

(ii) Decreasing the grazing angle and thus decreasing the variation of the heat load on the surface accordingly (reduces thermal mapping).

(iii) Using a longer substrate such that the central cone of relevant photons is located further away from the edges of the substrate, thus reducing the influence of end effects.

(iv) Decrease the absorbed power density by reducing the beam power upstream, e.g. by filters.

\section{Acknowledgements}

The authors thank Amparo Vivo and Amandine Chareyre for helpful discussions concerning typical state-of-the-art polishing slope errors and slope errors caused by mechanical clamping. Further, we would like to thank our colleagues from the optics group, Ray Barrett and his team as well as Robert Baker, for the help over the past years in our collaboration in projects covering multilayer mirror systems design at the ESRF.

\section{References}

Baker, R., Barrett, R., Clavel, C., Dabin, Y., Eybert-Berard, L., Mairs, T., Marion, P., Mattenet, M., Zhang, L., Baboulin, D. \& Guillemin, J. (2013). J. Phys. Conf. Ser. 425, 052015.

Biasci, J., Bouteille, J., Carmignani, N., Chavanne, J., Coulon, D., Dabin, Y., Ewald, F., Farvacque, L., Goirand, L., Hahn, M., Jacob, J., LeBec, G., Liuzzo, S., Nash, B., Pedroso-Marques, H., Perron, T., Plouviez, E., Raimondi, P., Revol, J., Scheidt, K. \& Serrière, V. (2014). Synchrotron Radiat. News, 27(6), 8-12.

Boley, B. A. \& Weiner, J. H. (1997). Theory of Thermal Stresses. New York: Dover Publications.

Brumund, P., Reyes-Herrera, J., Detlefs, C., Morawe, C., Sanchez del Rio, M. \& Chumakov, A. I. (2021). J. Synchrotron Rad. 28, 91-103.

Castro, A. R. B. de \& Reininger, R. (1991). Nucl. Instrum. Methods Phys. Res. A, 307, 135-141.

Chareyre, A. \& Brumund, P. (2021). Private communication.

Chumakov, A. I., Sergeev, I., Celse, J.-P., Rüffer, R., Lesourd, M., Zhang, L. \& Sánchez del Río, M. (2014). J. Synchrotron Rad. 21, 315-324.

Cocco, D., Hardin, C., Morton, D., Lee, L., Ng, M. L., Zhang, L., Assoufid, L., Grizolli, W., Shi, X., Walko, D. A., Cutler, G., Goldberg, K. A., Wojdyla, A., Idir, M., Huang, L. \& Dovillaire, G. (2020). Opt. Express, 28, 19242.

Cutler, G., Cocco, D., DiMasi, E., Morton, S., Sanchez del Rio, M. \& Padmore, H. (2020). J. Synchrotron Rad. 27, 1131-1140.

DiGennaro, R., Gee, B., Guigli, J., Hogrefe, H., Howells, M. \& Rarback, H. (1988). Nucl. Instrum. Methods Phys. Res. A, 266, 498506.

Khounsary, A. M. (1999). Proc. SPIE, 3773, 78-87.

Khounsary, A. M. \& Yun, W. (1996). Rev. Sci. Instrum. 67, 3354.

Kingslake, R. (1994). Opt. Photon. News, 5, 20-23.

Knopp, J., Fisher, M., Liu, Z., Maser, J., Reininger, R. \& Shi, X. (2018). Proceedings of the 10th Mechanical Engineering Design of Synchrotron Radiation Equipment and Instrumentation
(MEDSI2018), 25-29 June 2018, Paris, France, pp. 238-240. WEPH16.

Kutsal, M., Bernard, P., Berruyer, G., Cook, P. K., Hino, R., Jakobsen, A. C., Ludwig, W., Ormstrup, J., Roth, T., Simons, H., Smets, K., Sierra, J. X., Wade, J., Wattecamps, P., Yildirim, C., Poulsen, H. F. \& Detlefs, C. (2019). Mater. Sci. Eng. 580, 012007.

Li, Y., Khounsary, A. M. \& Nair, S. (2004). Proc. SPIE, 5533, 157.

Ludwig, W., King, A., Reischig, P., Herbig, M., Lauridsen, E., Schmidt, S., Proudhon, H., Forest, S., Cloetens, P., Roscoat, S. R., Buffière, J., Marrow, T. \& Poulsen, H. (2009). Mater. Sci. Eng. A, 524, 69-76.

Mattenet, M., Abernathy, D., Zontone, F., Detlefs, C., Grübel, G., Facchini, M. \& Jacquot, P. (2001). Nucl. Instrum. Methods Phys. Res. A, 467-468, 305-308.

Mattenet, M. \& Marot, G. (1996). Proc. SPIE, 2855, 180-186.

Menthel, U., Peatman, W. B. \& Senf, F. (1992). Rev. Sci. Instrum. 63, 481-484.

Middelmann, T., Walkov, A., Bartl, G. \& Schödel, R. (2015). Phys. Rev. B, 92, 174113 .

Morawe, C. (2019). AIP Conf. Proc. 2054, 060002.

Morawe, C. \& Osterhoff, M. (2010). X-ray Opt. Instrum. 2010, 479631.

Morawe, C., Peffen, J.-C., Ziegler, E. \& Freund, A. K. (2001). Proc. SPIE, 4145, 61-71.

Onuki, H. \& Elleaume, P. (2003). Editors. Undulators, Wigglers, and their Applications. pp. 75-79. London, New York: Taylor \& Francis.

Poulsen, H. F. (2012). J. Appl. Cryst. 45, 1084-1097.

Raimondi, P. (2016). Proceedings of International Particle Accelerator Conference (IPAC'16), 8-13 May 2016, Busan, Korea, pp. 20232027. WEXA01.

Rebuffi, L. \& Sanchez del Rio, M. (2017). Advances in Computational Methods for X-ray Optics IV, edited by K. Sawhney \& O. Chubar, p. 28. SPIE.

Rebuffi, L., Shi, X., Sanchez del Rio, M. \& Reininger, R. (2020). J. Synchrotron Rad. 27, 1108-1120.

Sanchez del Rio, M. \& Dejus, R. J. (2011). Proc. SPIE, 8141, 814115.

Sanchez del Rio, M., Wojdyla, A., Goldberg, K. A., Cutler, G. D., Cocco, D. \& Padmore, H. A. (2020). J. Synchrotron Rad. 27, 11411152.

Simons, H., King, A., Ludwig, W., Detlefs, C., Pantleon, W., Schmidt, S., Stöhr, F., Snigireva, I., Snigirev, A. \& Poulsen, H. F. (2015). Nat. Commun. 6, 6098.

Susini, J. (1995). Opt. Eng. 34, 361-376.

Tavares, P. F., Al-Dmour, E., Andersson, Å., Cullinan, F., Jensen, B. N., Olsson, D., Olsson, D. K., Sjöström, M., Tarawneh, H., Thorin, S. \& Vorozhtsov, A. (2018). J. Synchrotron Rad. 25, 1291-1316.

Touloukian, Y., Powell, R., Ho, C. \& Klemens, P. (1971). Thermophysical Properties of Matter, Vol. 1, Thermal Conductivity Metallic Elements and Alloys. Defense Technical Information Center.

Vivo, A. \& Barrett, R. (2021). Private communication.

Willmott, P. (2019). An Introduction to Synchrotron Radiation: Techniques and Applications, 1st ed. New York: Wiley.

Windt, D. L. (1998). Comput. Phys. 12, 360.

Yildirim, C., Cook, P., Detlefs, C., Simons, H. \& Poulsen, H. F. (2020). MRS Bull. 45, 277-282.

Zhang, L., Barrett, R., Cloetens, P., Detlefs, C. \& Sanchez del Rio, M. (2014). J. Synchrotron Rad. 21, 507-517.

Zhang, L., Barrett, R., Friedrich, K., Glatzel, P., Mairs, T., Marion, P., Monaco, G., Morawe, C. \& Weng, T. (2013). J. Phys. Conf. Ser. 425, 052029 .

Zhang, L., Lee, W.-K., Wulff, M. \& Eybert, L. (2003). J. Synchrotron Rad. 10, 313-319.

Ziegler, E. (1995). Opt. Eng. 34, 445. 